\title{
Variable Number Tandem Repeats for Global Strain Typing and Strain Differentiation of Mycobacterium leprae
}

\author{
Sanatosh Chokkakula ${ }^{1^{*}}$, Suliman S. Abdelrawaf ${ }^{2}$, Idress Hamad Attitalla ${ }^{2^{*}}$ and Ahmed A. Mehdi ${ }^{2}$ \\ ${ }^{1}$ School of Life and Health Sciences, Adikavi Nannaya University, Rajahmundry-535105, A.P, India \\ 2Omar Al-Mukhtar University, Faculty of Science, Microbiology Department, Box 919, Al-Bayda, Libya
}

*Corresponding author: Idress Hamad Attitalla, Omar Al-Mukhtar University, Faculty of Science, Microbiology Department, Box 919, Al-Bayda, Libya, E-mail: Idressttitalla2004@yahoo.com; biochemsanthu@gmail.com

Rec Date: July 22, 2014, Acc date: Aug 29, 2014, Pub date: Aug 31, 2014

Copyright: @ 2014 Attitalla IH, et al. This is an open-access article distributed under the terms of the Creative Commons Attribution License, which permits unrestricted use, distribution, and reproduction in any medium, provided the original author and source are credited.

\begin{abstract}
Leprosy is chronic granulomatous disease caused by Mycobacterium leprae and associated with the disability, stigma and discrimination to the affected individuals. Though often considered a disease of antiquity, it is found most commonly today in tropical and sub-tropical regions. Global efforts to eradicate leprosy have been largely successful in controlling its spread. Despite these efforts, the disease remains endemic countries emphasizing the need for greater scrutiny of its epidemiology. Strain typing and strain differentiation by Variable Number Tandem repeats (VNTR) could be useful in tracing origins and routes of infection, general leprosy surveillance and prevalence. Strain typing of Mycobacterium leprae by VNTR has been successfully carried out and predominance of leprosy bacilli in different geographical region also done by VNTR. In the present review, we reported global distribution $M$. leprae by Variable Number tandem repeats in systematic way to easy understanding of leprosy distribution.
\end{abstract}

\section{Review}

Leprosy is chronic granulomatous disease caused by Mycobacterium leprae and associated with the disability, stigma and discrimination to the affected individuals [1]. Though often considered a disease of antiquity, it is found most commonly today in tropical and sub-tropical regions [2]. Global efforts to eradicate leprosy have been largely successful in controlling its spread. Despite these efforts, the disease remains endemic countries, with 232,857 cases reported cases globally in 2012 [3]. India accounted for 1.27 of cases reported in 2011-2012, emphasizing the need for greater scrutiny of its epidemiology. strain typing and strain differentiation are very helpful to identify the source of infection, transmission of infection and spreading of disease, differentiating cases of relapse from re infection, and for unravelling possible links between human and nonreservoir sources [4,5]. In recent years molecular typing methodologies have complemented conventional infectious disease.

Strain tying and strain differentiation is a method which useful to distinguishing members of the same microbial species from one another on the basis of genotype. Strain typing explains that one isolate is same or it's different. Several molecular typing methods have been employed to distinguish the $M$. leprae strains over the years. Initially methods like surface antigen typing [6], multi-locus enzyme electrophoresis (MLEE) [7] and phage typing [8] were used for strain typing. DNA based strain typing methods were started from 1990. The methods includes fragment length polymorphism (RFLP) typing at locus such as rRNA operons [9] fingerprinting by randomly primed PCR [10] pulsed-field gel electrophoresis (PFGE), multi-locus sequence typing (MLST) [11], variable umber tandem repeats (VNTR) and SNP. If genetic diversity less, VNTR is good source for the identification of strain variation $[12,13]$. VNTRs have short, tandemly repeated motifs typically ranging from 1 to $60 \mathrm{bp}$ in length. Polymorphisms at those sites consist of differences in the number of repeat sequences contained by a VNTR and are the consequence of mutations which occur during DNA replication, when repeats are inserted or deleted by DNA polymerase due to slipped-strand mispairing [14]. There is considerably more variation in repeat numbers at VNTR locus than in non-repetitive DNA sequences, because length-altering mutations due to slipped-strand mispairing occur at a much higher rate than the inherent DNA substitution or mutation frequency of DNA polymerase [15].

Surprisingly little is known about the biology and epidemiology $M$. leprae. Two key properties of the organism explain both much of our ignorance about the organism and the fairly primitive state of the molecular epidemiology of $M$. leprae. In most of the bacteria small sequence of few housekeeping genes as in MLST is enough for inferring relationships among strains. But genetically monomorphic species reveals that no genetic polymorphism in these species $[16,17]$. $M$. leprae has associated with the less genetic diversity. This less genetic diversity due to high content of pesudogenes and several DNA repair genes [18]. Sever notable issues have been identified which limit the molecular epidemiological study of leprosy. Good efforts were made to strain typing even within less genetic polymorphism genome by restriction fragment length polymorphism (RFLP) [19,20], examination of dispersed repeats [21] and sequence analysis of variable genomic regions [22] proved universally unsuccessful but last decades powerful methods have been made to strain typing of leprosy.

The actual strain typing methods were started after complete genome sequence of $M$. leprae TN strain and initially 50 VNTR loci have identified. Continue efforts on this VNTR studies showed the path for utility in molecular epidemiology studies for strain typing [23-26]. Robust PCR amplification technique have involved in the development and validation of VNTR markers. To date, based on several techniques, several VNTR markers were addressed and in future several VNTR loci may add. Initial studies of VNTR have associated with several controversies which were later solved by subsequent VNTR studies [27]. Whole genome sequencing methods 
were dropped as it is cost effective so that alternative studies have made for strain typing in which SNP and VNTRs are most effective for leprosy. Although several polymorphic sites were available, only small fraction regions were associated with variation and those were involved in the global strain typing of M.leprae. The information provided by VNTR neither was nor did similar to the SNP result and resolution of strain relationship with SNP quite limit. So VNTR study was suitable for strain typing of microorganisms.

The selection of VNTR was mainly dependent on timescale of the event which can easily tracing strain variation within short time. These VNTR markers were also more suitable for probing historical distribution of strains over hundreds to thousands of years. Molecular epidemiology studies of $M$. leprae strains collected within individual nations have revealed several general properties of leprosy and leprosy transmission at that population level. After standardization VNTR PCR study in proper way by Gillis in the year 2009, several studies were used those primers for study of strain variation globally [26,28-31]. These studies indicated that, the strain possess same allelic distribution with the country and those strains have some alleles which were alike of the other countries in allelic distribution which indicated that those are genetically similar and migration was there in these populations. Some studies have strongly suggested that leprosy bacilli strains were migrated from India to Philippines with highest genetic diversity and identified 3 distinct groups. Interestingly the first sequenced Indian strain TN was genetically similar with Philippines leprosy strains which suggested that $\mathrm{TN}$ is not an Indian strain and which may emigrate from some other countries. The detection rate of new and relapse cases in each year and the national prevalence reflects the continuing spread of leprosy. In such areas the strain typing and strain differentiation are very helpful to identify the source of infection, transmission of infection and spreading of disease, differentiating cases of relapse from re infection, and for unravelling possible links between human and non-reservoir sources.

In recent years molecular typing methodologies have complemented conventional infectious disease. With the publication in 2001 of the complete genome sequence of an isolate from Tamil Naidu, India called TN strain; selection of potential polymorphic genomic markers for strain typing was feasible. This contained short tandem repeats (STR) regions that had potential for genetic polymorphism by expansion or contraction of repeats and therefore for strain typing of $M$. leprae. A list of loci were targeted for strain typing $[32,33]$ and multi locus variable number tandem repeat analysis (MLVA) as means for molecular differentiation of $M$. leprae, within and amongst leprosy patients emerged [23] . Globally several studies using one or more loci have been published for isolates from Colombia, Brazil, Philippines, Thailand and China $[26,29,30]$. The TN sequence also served as template for the identification of single nucleotide polymorphism (SNPs) by comparison with genome sequence of a few other reference isolates. Three SNPs which yielded only four patterns (SNP1-4) and are further classified into 16 subtypes designated as SNP1A-D, SNP2F-H, SNP3I-M and SNP4N-P. The relative merits and information that can be generated from polymorphism at these two types genetic markers (STR \&SNPs) have been databased [34]. The genetic diversity of $M$.leprae is very low which was successfully typed basing on short tandem repeats (STR) or microsatellites (repeat length 2-5 bp) and minisatellite (repeat length 6-50 bp) called as VNTR. In the present review we focused on the VNTR allele distribution in globally.
The first strain typing analysis of Mycobactermberium leprae by variable number tandem repeats have been carried out by taking strains of leprosy patients and passes to Armadillos. This study have analysed copy number of (AT)17, (C)20, (CG)6, 6-7,12-5,18-8,21-3,27-5 and (AGA)20 (Nathan et al., 2004). The study taking strains as Thai-53, Kyoto-1, Zensho-4, and Korea3-2, and 17 samples, namely, the fourth generation of Thai-53 (Thai-53 4th), Thai-53 7th, Thai-53 11th, Kyoto-1 3rd, Kyoto-1 5th, Kyoto-1 7th, Kyoto-1 8th, Zensho-4 (biopsy specimen), Zensho-4 1st, Zensho-4 2nd, Zensho-4 3rd, Zensho-4 4th, Korea3-2 (biopsy specimen), Korea3-2 1st, Korea3-2 2nd, Korea3-2 3rd, and Korea3-2 4th for allelic variation of (T)8(A)6 (T)6(N)7(T)8 (A)9 (G)9 (C)9 (G)10a (G)10b (G) 11 (G)12 (C)16(G)8 (C)20 (G)22 (CG)6 (AC)8a (AC)8b (AC)9

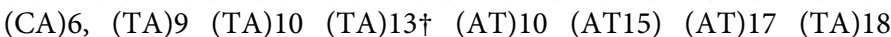
$\begin{array}{lllll}(\mathrm{ACC}) 5 & \text { (GGT)5 (AGT)5a } & \text { (AGT)5b (ACT)5 } & \text { (GTA)9 } & \text { (AGA)20 }\end{array}$ (CACCG) 3 loci and these results demonstrated that these strains contain highest number $8,6,6,7,8,8,9$ $10,10,11,10,9,14,7,10,14,6,9,8,7,6,8,12,19,8,14,13,17,5,4,5,5,5,11,11$ and 3 for loci of (T)8,(A)6, (T)6,(N)7,(T)8, (A)9, (G)9, (C)9, (G)10a, (G)10b，(G)11，(G)12，(C)16,(G)8，(C)20，(G)22，(CG)6，(AC)8a, (AC)8b, (AC)9, (CA)6, (TA)9, (TA)10, (TA)13, (AT)10, (AT15), (AT)17, (TA)18, (ACC)5, (GGT)5, (AGT)5a, (AGT)5b, (ACT)5, (GTA)9, (AGA) 20 and (CACCG) 3 respectively [35]. Later, in 2007 one study conducted for molecular typing of $M$. leprae by taking new leprosy cases from Qiubei with (AC)9, (AC)8a, 6-7, (GAA)21, (GTA)9, (AT)17, (AT) 15 and (TA)18. Strain typing of $M$. leprae by (TTC) 21 , (GTA)9, (AT)14, (AT)15 and (AT)17 loci have been done from the strains of Mali and results showed that 14,9, 14, 13 and 13 copy number has most predominant [21].

Until 2009, the strain typing method was mainly by RFLP but the actually strain typing methods in leprosy based on VNTR was mainly started in the year 2009. In the year 2009, extensive research has been done on strain typing of leprosy based on different loci. A single isolate of M. leprae (NHDP60) was passaged serially ( $\mathrm{H} 1, \mathrm{H} 2$ and $\mathrm{H} 3$ ) through three armadillos following the first propagation cycle $(\mathrm{H} 0)$ in an armadillo for a total propagation time of 5 years and 7 months. The passage times were $\mathrm{H} 0,31$ months; $\mathrm{H} 1,12$ months; $\mathrm{H} 2,11$ months and H3, 13 months. M. leprae-infected nodules or lymph node tissue from each passage was processed for DNA extraction using the DNeasy Kit and these was subjected to VNTR typing. This is the first report which gave clear cut idea of strain typing. In 2009 itself, Cebu, Philippines leprosy patients have strain typed and predominant copy number $8,9,5,15,3,9,14,9,5,7,21,24,8,4$ and 2 for loci (AC)8b, (GTA) 9 , (GGT)5, (AT)17, 21-3, (AC)9, (AT)15, (AC)8,a 27-5, 6-7, (TA)18, (TTC)21, $18-8,12-5$ and 23-3 respectively [24]. From Thailand leprosy strains, the prominent copy number $7,9,4,13,2.7,13,9,5,6,11,20,4$ and 2 have been identified for the loci (AC)8b, (GTA)9, (GGT)5, (AT)17, 21-3, (AC) 9, (AT)15, (AC)8a, 27-5, 6-7, (TA)18, (GAA)21, 12-5 and 23-3 respectively.

The loci (AC)8b, (GTA)9, (GGT)5, (AT)17, 21-3, (AC)9, (AT)15, (AC) 8a, 27-5, 6-7,, (TA)18, (GAA)21, 18-8, 12-5, 23-3, (TA)10 from the Philippians leprosy patients have associated with predominant copy number $8,9,5,15,3,9,14,9,5,7,17,25,8,4,2,11$ and 3 respectively [30]. From the Colombia, the copy number $7,8,4,12,2,8,14,8,4,6,17,11,8,4,2,4$ was (AC)8b, (GTA)9, (GGT)5, (AT) 17, 21-3, (AC)9, (AT)15, (AC)8a, 27-5, 6-7, (TA)18, (GAA)21, 18-8, 12-5 and 23-3 respectively (Nora et al.,2009). In China, (AC)8b, (GTA)9, (GGT)5, (AT)17, rpoT, 21-3, (AC)9, (AT)15, (AC)8a, 27-5, 6-7, (TA)18, (GAA)21, 18-8, 23-3, 12-5 and (TA)10 loci have copy number of $8,9,4,13,3,2,8,16,10,5,8,15,22,7,2,3,10$ as predominant [26]. 
In 2009, the Brazilian leprosy patients were strain typed with (AC)8b (GTA)9 (GGT)5 (AT)17 21-3 (AC)9 (AT)15 (AC)8a 27-5 6-7 (TA)18 (GAA) $21 \quad$ (TA) $10 \quad 23-3 \quad 12-5 \quad 18-8 \quad$ loci and reported that $7,11,4,4,2,8,10,8,4,6,14,11,6,2,4$ and 8 copy number as a higher.

Several studies have been described for strain typing of leprosy by VNTR to understanding strain distribution and allelic distributions of Indian leprosy patients. The report from South India stated that, the predominant copy number $7,9,4,11,3,2,8,13,8,2,5,6,16,15,7,4,2$ and 8 have been associated with loci (AC)8b, (GTA)9, (GGT)5, (AT)17, 6-3a, 21-3, (AC)9, (AT)15, (AC)8a, ML, 27-5, 6-7, (TA)18, (GAA)21, $18-8,12-5,23-3$ and (TA) 10 respectively. Variation in (TTC) 21 repeats in Lepromatous Leprosy cases have done from strains of Andhra Pradesh and Odisha and results stated that 12 copy number was predominant for (TTC)21 loci [36]. Like South India leprosy patients, North India and Mumbai leprosy patients were also strain typed and these results were somewhat resembles with the South Indian leprosy patients $[37,38]$.

\section{References}

1. Scollard DM, Adams LB, Gillis TP, Krahenbuhl JL, Truman RW, et al. (2006) The continuing challenges of leprosy. Clin Microbiol Rev 19: 338-381.

2. Britton WJ, Lockwood DN (2004) Leprosy. Lancet 363: 1209-1219.

3. WHO report 2012.

4. Linder K, Zia M, Kern WV, Pfau RK, Wagner D (2008) Relapses vs. reactions in multibacillary leprosy: proposal of new relapse criteria. Trop Med Int Health 13: 295-309.

5. Shetty VP, Wakade AV, Ghate SD, Pai VV, Ganapati RR, et al. (2005) Clinical, histopathological and bacteriological study of 52 referral MB cases relapsing after MDT. Lepr Rev 76: 241-252.

6. Lancefield RC (1928) The Antigenic Complex Of Streptococcus Haemolyticus : I. Demonstration Of A Type-Specific Substance In Extracts Of Streptococcus Haemolyticus. J Exp Med 47: 91-103.

7. Milkman R (1973) Electrophoretic variation in Escherichia coli from natural sources. Science 182: 1024-1026.

8. Ahmed R, Bopp C, Borczyk A, Kasatiya S (1987) Phage-typing scheme for Escherichia coli O157:H7. J Infect Dis 155: 806-809.

9. Low DA, Braaten BA, Ling GV, Johnson DL, Ruby AL (1988) Isolation and comparison of Escherichia coli strains from canine and human patients with urinary tract infections. Infect Immun 56: 2601-2609.

10. Welsh J, McClelland M (1990) Fingerprinting genomes using PCR with arbitrary primers. Nucleic Acids Res 18: 7213-7218.

11. Maiden MC, Bygraves JA, Feil E, Morelli G, Russell JE, Urwin R, et al. (1998) Multilocus sequence typing: a portable approach to the identification of clones within populations of pathogenic microorganisms. Proc. Natl. Acad. Sci USA 95: 3140-3145.

12. Keim P, Price LB, Klevytska AM, Smith KL, Schupp JM, et al. (2000) Multiple-locus variable-number tandem repeat analysis reveals genetic relationships within Bacillus anthracis. J Bacteriol 182: 2928-2936.

13. Klevytska AM, Price LB, Schupp JM, Worsham PL, Wong J, et al. (2001) Identification and characterization of variable-number tandem repeats in the Yersinia pestis genome. J Clin Microbiol 39: 3179-3185.

14. Streisinger G, Okada Y, Emrich J, Newton J, Tsugita A, Terzaghi E, et al. (1966) Frame shift mutations and the genetic code. This paper is dedicated to Professor Theodosius Dobzhansky on the occasion of his 66th birthday. Cold Spring Harb. Symp. Quant. Biol 31: 77-84.

15. Yamada NA, Smith GA, Castro A, Roques CN, Boyer JC, Farber RA et al. (2002) Relative rates of insertion and deletion mutations in dinucleotide repeats of various lengths in mismatch repair proficient mouse and mismatch repair deficient human cells. Mutat. Res 499: 213-225.
16. Achtman M (2008) Evolution, population structure, and phylogeography of genetically monomorphic bacterial pathogens. Annu Rev Microbiol 62: 53-70.

17. Comas I, Homolka S, Niemann S, Gagneux S (2009) Genotyping of genetically monomorphic bacteria: DNA sequencing in Mycobacterium tuberculosis highlights the limitations of current methodologies. PLoS One 4: e7815.

18. Cole ST, Eiglmeier K, Parkhill J, James KD, Thomson NR, Wheeler PR, et al. (2001) Massive gene decay in the leprosy bacillus. Nature 409: 10071011.

19. Clark-Curtiss JE, Docherty MA (1989) A species-specific repetitive sequence in Mycobacterium leprae DNA. J Infect Dis 159: 7-15.

20. Williams DL, Gillis TP, Booth RJ, Looker D, Watson JD (1990) The use of a specific DNA probe and polymerase chain reaction for the detection of Mycobacterium leprae. J Infect Dis 162: 193-200.

21. Woods SA, Cole ST (1990) A family of dispersed repeats in Mycobacterium leprae. Mol Microbiol 4: 1745-1751.

22. de Wit MY, Klatser PR (1994) Mycobacterium leprae isolates from different sources have identical sequences of the spacer region between the 16S and 23S ribosomal RNA genes. Microbiology $140: 1983-1987$.

23. Groathouse NA, Rivoire B, Kim H, Lee H, Cho SN, et al. (2004) Multiple polymorphic loci for molecular typing of strains of Mycobacterium leprae. J Clin Microbiol 42: 1666-1672.

24. Kimura M, Sakamuri RM, Groathouse NA, Rivoire BL, Gingrich D, Krueger- Koplin S, et al. (2009) Rapid variable-number tandem-repeat genotyping for Mycobacterium leprae clinical specimens. J. Clin. Microbiol 47: 1757-1766.

25. Truman R, Fontes AB, De Miranda AB, Suffys P, Gillis T (2004) Genotypic variation and stability of four variable-number tandem repeats and their suitability for discriminating strains of Mycobacterium leprae. J Clin Microbiol 42: 2558-2565.

26. Xing Y, Liu J, Sakamuri RM, Wang Z, Wen Y, et al. (2009) VNTR typing studies of Mycobacterium leprae in China: assessment of methods and stability of markers during treatment. Lepr Rev 80: 261-271.

27. Gillis T, Vissa V, Matsuoka M, Young S, Richardus JH, et al. (2009) Characterisation of short tandem repeats for genotyping Mycobacterium leprae. Lepr Rev 80: 250-260.

28. Cardona-Castro N, Beltrán-Alzate JC, Romero-Montoya IM, Meléndez E, Torres F, et al. (2009) Identification and comparison of Mycobacterium leprae genotypes in two geographical regions of Colombia. Lepr Rev 80: 316-321.

29. Nogueira A, Fontes B, Sakamuri RM (2009) Genetic diversity of Mycobacterium leprae isolates from Brazilian leprosy patients. Lepr Rev 80: $302-315$

30. Sakamuri RM, Harrison J, Gelber R, Saunderson P, Brennan PJ, et al. (2009) A continuation: study and characterisation of Mycobacterium leprae short tandem repeat genotypes and transmission of leprosy in Cebu, Philippines. Lepr Rev 80: 272-279.

31. Srisungnam S, Rudeeaneksin J, Lukebua A, Wattanapokayakit S, Pasadorn S, Mahotarn K, et al. (2009) Molecular epidemiology of leprosy based on VNTR typing in Thailand. Lepr Rev 80: 280-289.

32. Shin YC, Lee H, Lee H, Walsh GP, Kim JD, et al. (2000) Variable numbers of TTC repeats in Mycobacterium leprae DNA from leprosy patients and use in strain differentiation. J Clin Microbiol 38: 4535-4538.

33. Matsuoka M, Maeda S, Kai M, Nakata N, Chae GT, et al. (2000) Mycobacterium leprae typing by genomic diversity and global distribution of genotypes. Int J Lepr Other Mycobact Dis 68: 121-128.

34. Monot M, Honore N, Baliere C, Ji B, Sow S, Brennan PJ, et al. (2008) Are Variable-Number Tandem Repeats Appropriate for Genotyping Mycobacterium leprae?. Journal of Clinical Microbiology 46: 2291-2297.

35. Zhang L, Budiawan T, Matsuoka M (2005) Diversity of potential short tandem repeats in Mycobacterium leprae and application for molecular typing. J Clin Microbiol 43: 5221-5229.

36. Chokkakula S, Dasari K, Attitalla IH, Aparna S, Ponnada P, Male MM, et al. (2014) Strain Typing and Strain Differentiation of Mycobacterium 
Citation: Chokkakula S, Abdelrawaf SS, Attitalla IH, Mehdi AA (2014) Variable Number Tandem Repeats for Global Strain Typing and Strain Differentiation of Mycobacterium leprae. J Med Microb Diagn 3: 156. doi:10.4172/2161-0703.1000156

Page 4 of 4

leprae by TTC Repeats. International Journal of Pharmacology 10 168-174.

37. Lavania M, Katoch K, Sharma R, Sharma P, Das R, et al. (2011)

Molecular typing of Mycobacterium leprae strains from northern India using short tandem repeats. Indian J Med Res 133: 618-626.
38. Kuruwa S, Vissa V, Mistry N (2012) Distribution of Mycobacterium leprae strains among cases in a rural and urban population of Maharashtra, India. J Clin Microbiol 50: 1406-1411. 